\title{
ESTUDO NUMÉRICO DA INTERAÇÃO ENTRE CONVECÇÃO RASA E RADIAÇÃO COM ÊNFASE NO CICLO DIURNO DO BALANÇO DE ENERGIA À SUPERFÍCIE NA AMAZÔNIA
}

\author{
ENIO PEREIRA DE SOUZA ${ }^{1}$, ZILURDES FONSECA LOPES ${ }^{1,2}$, TACIANA LIMA ARAUJO ${ }^{1}$ \\ ${ }^{1}$ Universidade Federal de Campina Grande (UFCG), Campina Grande-PB, \\ ${ }^{2}$ Fundação Cearense de Meteorologia e Recursos Hídricos (FUNCEME), Fortaleza-CE. \\ esouza@dca.ufcg.edu.br, tacianalimaraujo@hotmail.com, zilurdes@gmail.com
}

Recebido Abril 2008 - Aceito Fevereiro 2009

\begin{abstract}
RESUMO
Estuda-se neste artigo o efeito da convecção rasa no ciclo diurno do balanço de energia à superfície na Amazônia. $\mathrm{O}$ enfoque foi dado ao papel do sombreamento, devido aos cúmulos rasos na configuração do forçamento convectivo à superfície. Um esquema para o cálculo da cobertura de cúmulos rasos foi implementado no modelo BRAMS. Foram executados dois experimentos: um com convecção rasa e sem interação com a radiação, e o segundo com convecção rasa e interação com a radiação. Os resultados mostraram que a consideração da interação entre convecção rasa e radiação produz simulações mais realistas. Isso foi mais pronunciado por volta do meio-dia local e início da tarde, quando os cúmulos rasos estão com sua atividade máxima. Os fluxos e a cobertura foram bastante sensíveis às variações no forçamento radiativo. Apesar da melhoria da qualidade dos fluxos, do ponto de vista qualitativo, ficou evidente a deficiência do modelo em representar esses fluxos nas primeiras e últimas horas do ciclo diurno.

Palavras-chave: convecção rasa, radiação, BRAMS.
\end{abstract}

\begin{abstract}
A NUMERIC STUDY OF THE INTERACTION BETWEEN SHALLOW CONVECTION AND RADIATION WITH EMPHASIS IN THE DIURNAL CYCLE OF THE SURFACE ENERGY BUDGET IN AMAZONIA

The effect of shallow convection on the diurnal cycle of the surface energy budget is investigated. Focus is on the role of the cloud shading on the establishment of the surface convective forcing. A scheme for calculating the shallow cumulus cover was implemented in the BRAMS atmospheric model. Two sets of experiments were performed: in the first experiment, the model had no interaction between shallow convection and radiation, and in the second type of experiment shallow convection interacts with radiation. Results show that the consideration of the interaction between shallow cumulus and radiation leads to more realistic simulations. This is more pronounced around the local noon and early afternoon, when the shallow cumulus activity reaches a maximum. The surface fluxes and the cumulus cover were very sensitive to the variations in the radiative forcing. Even though, from a qualitative viewpoint, there is an improvement on the quality of the simulated fluxes, it became evident a model flaw in representing these fluxes during the first hours of the diurnal cycle.
\end{abstract}

Keywords: shallow convection, radiation, BRAMS. 


\section{INTRODUÇÃO}

O forçamento primário para os processos atmosféricos resulta da diferença entre a energia absorvida e a emitida pelo sistema terra-atmosfera. A física envolvida no processo pode ser encontrada em qualquer livro-texto de radiação (e.g., Liou, 1980). A partição da energia entre calor sensível e calor latente, determina a energia disponível para a evaporação da água da superfície, para a transpiração das plantas e para elevar ou diminuir a temperatura da superfície e do ar. As nuvens convectivas são componentes centrais para a compreensão do tempo e do clima. Elas não somente transportam calor e umidade verticalmente na atmosfera, mas também afetam fortemente o balanço de energia nas escalas local e global. Os cúmulos profundos, que têm escalas entre $10^{2}$ e $10^{3} \mathrm{~m}$, são responsáveis por elevadas taxas de precipitação e, historicamente, têm recebido mais desenvolvimento em modelos numéricos.

Apesar de os cúmulos rasos receberem menos atenção em modelos numéricos, representam um importante papel para a manutenção da estrutura do sistema terra-atmosfera. Além disso, a transição da convecção de rasa para profunda é elemento-chave para uma boa representação do ciclo diurno da precipitação (Khairoutdinov e Randall, 2003, e Grabowski et al., 2006). Eles atuam como uma fonte de calor e um sumidouro de umidade na parte superior da camada de mistura, causando aquecimento e secagem na região em contorno da base da nuvem. Devido às nuvens cúmulos rasos não produzirem precipitação [note-se a diferença entre as nuvens definidas em modelagem e as estudadas por Pereira e Rutledge (2006)], e pela presença de uma camada mais estável e seca acima da camada de cúmulos, a re-evaporação causa resfriamento e umedecimento da parte superior da nuvem, o que causa um transporte líquido de calor para baixo e de umidade para cima na região em que a convecção rasa atua (Betts, 1975). Como os cúmulos rasos têm escala típica da ordem de $1 \mathrm{~km}$, seus efeitos coletivos (aquecimento, resfriamento evaporativo, mistura, etc) não são determinados explicitamente nos modelos. Então esses efeitos são modelados através da parametrização. Souza (1999) propôs uma parametrização para a convecção rasa no modelo Regional Atmospheric Modelling System - RAMS, para calcular o fluxo de massa pela convecção durante o Rondonian Boundary Layer Experiment-3 - RBLE-3. Suas simulações representaram de forma mais realistas os perfis termodinâmicos da atmosfera.

Siebesma et al. (2003) mostraram a intercomparação da convecção cúmulos rasos em modelos de simulação de grandes turbilhões, e averiguaram que uma simulação de 6 horas teve excelente concordância da estrutura vertical termodinâmica observada de modelo para modelo. O estudo de Deng et al. (2003) apontou que os esquemas de convecção rasa podem simular ambientes sinóticos diferentes e as condições de mudanças bruscas associadas com fortes fluxos de superfície.

Por ser um processo intermediário entre a camada limite planetária e a atmosfera livre, a atividade dos cúmulos rasos está sujeita a vários mecanismos de retro-alimentação, que podem ser positivos ou negativos para a configuração do perfil de estabilidade local, com fortes implicações na dinâmica da circulação atmosférica. Stull (1985) enumerou alguns desses mecanismos: a retro-alimentação dinâmica implica que o movimento ascendente dentro da nuvem transporta uma perturbação negativa de entalpia e o movimento descendente, na periferia dos cúmulos, transporta uma perturbação positiva de entalpia. A retro-alimentação termodinâmica implica em aquecimento, devido à condensação, na região inferior da nuvem e resfriamento evaporativo na parte superior. A retro-alimentação radiativa implica em aquecimento na base, devido à absorção de radiação de onda longa, e resfriamento no topo, devido à emissão. Há várias alternativas para a incorporação desses efeitos, através de parametrização, em modelos numéricos (Betts e Miller, 1986; Tiedtke, 1989). Para o caso da radiação solar, o efeito mais importante é a atenuação da radiação que atinge a superfície, o que implica em impacto sobre os fluxos.

O objetivo deste trabalho é investigar o impacto da convecção rasa no ciclo diurno do balanço de energia à superfície na Amazônia. O enfoque é dado ao papel do sombreamento, devido aos cúmulos rasos na configuração do forçamento convectivo em superfície, utilizando como ferramenta o modelo Brazilian developments on the Regional Atmospheric Modeling System-BRAMS. Para isso, um esquema de parametrização da cobertura de cúmulos rasos foi implementado neste modelo. Na secção 2, a metodologia e os dados são apresentados; na secção 3 os resultados são discutidos e as considerações finais são apresentadas na secção 4 .

\section{MATERIAL E MÉTODOS}

\subsection{Descrição do modelo BRAMS}

O modelo numérico usado neste trabalho foi o "Brazilian developments on the Regional Atmospheric Modeling System" (BRAMS, http://brams.cptec.inpe.br), na versão 4.0, que foi desenvolvido a partir do RAMS (Regional Atmospheric Modeling System), cuja estrutura básica é descrita por Pielke et al. (1992). Dentre as parametrizações físicas utilizadas neste trabalho estão: radiação de onda longa e de onda curta de acordo com Chen e Cotton (1983). A turbulência na Camada Limite Planetária é calculada segundo Mellor e Yamada (1986). A microfísica segue o esquema descrito por Walko et al. (1995). A convecção profunda é do tipo Kuo (Kuo, 1974), modificada 
por Molinari (1985) e Molinari e Corsetti (1985). A interação superfície-atmosfera foi feita no BRAMS através do modelo LEAF-2 (Walko et al., 2000).

\subsection{Descrição do Esquema de Convecção Rasa}

O esquema proposto por Souza (1999) é do tipo fluxo de massa. O modelo de nuvens é do tipo Arakawa e Schubert (1974). O fluxo de massa baseia-se no seguinte princípio: Uma vez que a atmosfera é forçada pelo fluxo de energia à superfície, um fluxo de massa é necessário para que a atmosfera tente restabelecer seu perfil de equilíbrio radiativo-convectivo local. Isso estabelece uma relação direta entre o fluxo de massa convectivo e o forçante de superfície, reproduzindo uma característica observacional (Wilde et al., 1985).

A descrição completa do esquema pode ser encontrada em Souza e Silva (2003). Aqui é apresentado um breve resumo, pra facilitar a compreensão da discussão sobre a cobertura de nuvens. As equações prognósticas para entalpia "s" e umidade específica total “ $q_{t}$ ” são:

$$
\begin{gathered}
\frac{\partial \bar{s}}{\partial t}=-\frac{\partial}{\partial z} \overline{\left(w^{\prime} s_{l}^{\prime}\right)}=-\frac{\partial}{\partial z}\left(\frac{M_{c}}{\rho}\left(s_{c}-L q_{l c}-\bar{s}\right)\right) \\
\frac{\partial \bar{q}_{t}}{\partial t}=-\frac{\partial}{\partial z} \overline{\left(w^{\prime} q_{t}^{\prime}\right)}=-\frac{\partial}{\partial z}\left(\frac{M_{c}}{\rho}\left(q_{v_{c}}+q_{l c}-\bar{q}_{t}\right)\right)
\end{gathered}
$$

em que $s$ é a energia estática seca, $q_{t}$ é a umidade específica total, $M_{c}$ é o fluxo de massa convectivo, $q_{l c}$ e $q_{v c}$ são, respectivamente, a umidade especifica da água líquida e o vapor d'água, $p$ é a densidade do ar, L é o calor latente de vaporização. Os demais símbolos são os usuais.

O fluxo de massa convectivo é obtido por argumentos termodinâmicos. $\mathrm{O}$ raciocínio é de que os fluxos convectivos são substanciados a partir da conversão da energia absorvida em forma de calor da superfície em trabalho mecânico, de acordo com a teoria da máquina térmica (Rennó e Ingersoll, 1996). O fluxo de massa convectivo na base da nuvem é:

$$
M_{c}=\rho \sigma w_{c}=\frac{\eta F}{B}
$$

em que $\sigma$ é a área fracional, coberta por correntes convectivas, $\mathrm{w}_{\mathrm{c}}$ é a velocidade vertical convectiva, $\eta$ é a eficiência termodinâmica, $F$ é o fluxo de calor total de superfície e $B$ é o empuxo total das parcelas em convecção.

As propriedades da nuvem, caracterizadas pelo símbolo c, são obtidas a partir das equações de Arakawa e Schubert (1974):

$$
\begin{aligned}
& \frac{\partial h_{c}}{\partial z}=-\lambda_{z}\left(h_{c}-\bar{h}\right) \\
& \left(s_{c}-\bar{s}\right) \approx \frac{1}{1+\gamma_{v}}\left(h_{c}-\bar{h}_{s}\right) \\
& q_{v c}=\bar{q}_{s}+\frac{\gamma_{v}}{\left(1+\gamma_{v}\right) L}\left(h_{c}-\bar{h}_{s}\right)
\end{aligned}
$$

$$
\begin{aligned}
\text { nas quais, } \gamma_{v} & \equiv\left(\frac{L}{c_{p}} \frac{\partial q_{v s}}{\partial T}\right)_{p} \text { e } \\
\frac{\partial q_{k}}{\partial z} & =-\frac{\partial q_{v c}}{\partial z}=-\lambda_{z}\left(q_{v c}-\bar{q}_{v}\right)-\lambda_{z} q_{l c}
\end{aligned}
$$

em que $h_{c}$ é a energia estática úmida da nuvem e $\lambda_{z}$ é a taxa de entranhamento na nuvem.

O fluxo de massa, calculado pela Equação (3) é linearmente interpolado para zero no topo da nuvem.

\subsection{Parametrização da Cobertura de Nuvens Cúmulos}

Albrecht (1981) definiu o decaimento das nuvens pela diferença do teor de água total entre nuvem e o ambiente, ele também estabeleceu que a nuvem é aproximadamente não flutuante quando sua evaporação é completa. Em tal caso, para uma nuvem individual, a evolução temporal do teor de água dentro da nuvem é governada por uma função de decaimento exponencial com o tempo, dada por:

$$
\frac{d Q}{d t}=-\frac{Q}{\tau}
$$

em que, $Q=q_{v c}+q_{l c}-\bar{q}_{t}$ é a diferença do teor de água total entre a nuvem e o ambiente e $\tau$ é o tempo de decaimento exponencial. Baseado nesse princípio, Albrecht (1981), definiu que o campo total da cobertura de nuvem é:

$$
c=\frac{S R-1}{S R-R H}
$$

em que: $R H=\bar{q} / \bar{q}_{s}$ é a umidade relativa do ambiente e $S R=$ $\left(q_{c 0}+q_{l 0}\right) / \bar{q}_{s}$ é a razão de saturação. No entanto, essa equação não é aplicada para nuvens com empuxo negativo, nas quais SR seria menor que 1 , resultando em uma cobertura de nuvem negativa.

Para solucionar o problema acima, Haiden (1996), definiu uma nova razão de saturação (SR). Ele utilizou a energia 
estática úmida no processo de decaimento pelo balanço de calor, dada por: $h=c_{p} T+g z+L q$, em que: $\mathrm{z}$ é a altura, $\mathrm{g}$ aceleração da gravidade e $c_{p}$ é o calor específico do ar a pressão constante.

A evolução temporal da energia estática úmida é governada por uma equação análoga à Equação (8).

$$
\frac{d H}{d t}=-\frac{H}{\tau}
$$

em que: $H=h_{c}-\bar{h}$.

A manipulação dessas equações fornece uma expressão para a quantidade de nuvem total idêntica a da Equação (9). Porém, a razão de saturação é definida como $S R=1+\left(1+y_{v}\right)$. $q_{l 0} / \bar{q}_{s}$. Em termos do déficit de saturação do ambiente $\delta q$, a cobertura de nuvem é escrita na forma:

$$
c=\frac{1}{1+\delta \bar{q} /\left(1+\gamma_{v}\right) q_{l 0}}
$$

em que $q_{10}$ é a razão de mistura da água líquida inicial.

Os valores do conteúdo de água líquida (Equação 7) e de cobertura de nuvem (Equação 11) são passados para a rotina de radiação, que usa esses valores para aumentar a espessura óptica na camada de nuvem. Dessa forma, acontece a interação dos cúmulos rasos com a radiação de onda curta e de onda longa.

\subsection{Dados e Experimentos}

A simulação foi feita com duas grades, a primeira com resolução horizontal de $80 \mathrm{~km}$, cobrindo todo o território do Brasil e vizinhança e a segunda com $20 \mathrm{~km}$, centrada no estado de Rondônia. Foram considerados nove níveis do solo e dois experimentos foram realizados, o primeiro denominado de SHA

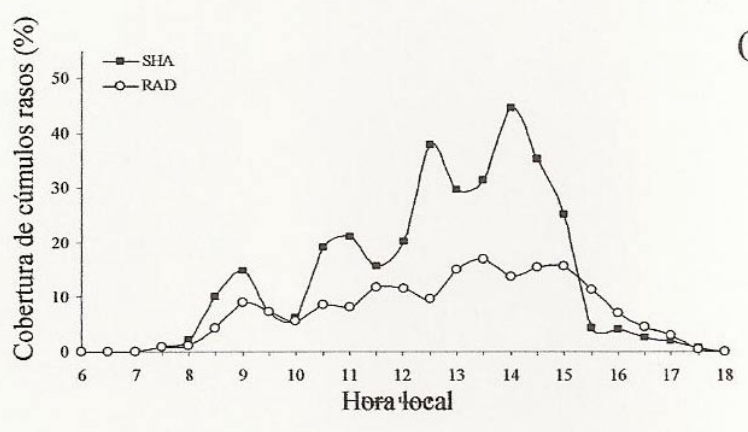

com convecção rasa e sem interação com a radiação solar, o segundo denominado de RAD, a convecção e a interação com a radiação foi ativada. As simulações para o regime de vento de oeste $\left(\mathrm{W}_{2}\right)($ Rickenbach et al., 2002) foram feitas para o período de três dias, entre 05 de Fevereiro a 00 UTC até 08 de Fevereiro a $00 \mathrm{UTC}$, e para o regime de leste $\left(\mathrm{E}_{3}\right)$ que compreende o período de 10 de Fevereiro a 00 UTC até 13 de Fevereiro a 00 UTC. Embora haja outros períodos de ventos característicos (Rickenbach et al., 2002), infelizmente somente para esses dois regimes de vento há dados observados, tanto de calor sensível quanto de calor latente, saldo de radiação, temperatura do ar com três dias consecutivos para fazer as comparações entre as simulações e as observações. Os dados observados são da campanha WETAMC/LBA-Wet Season Atmospheric Mesoscale Campaign do Experimento de Grande escala da biosferaAtmosfera na Amazônia (Silva Dias et al., 2002).

\section{RESULTADOS E DISCUSSÃO}

As figuras a seguir foram confeccionadas através da média horária (hora local de Rondônia) para cada regime de vento. As Figuras representam somente o ciclo diurno (6 às 18 horas local) das variáveis analisadas, uma vez que o modelo não simula a cobertura de nuvem no período noturno (nuvem gerada por turbulência mecânica). Os resultados analisados são os que se referem à grade de $20 \mathrm{~km}$, que é centrada no estado de Rondônia. Os valores correspondem à média de uma área de 1x1 grau em torno do ponto de medição.

A Figura 1a mostra os ciclos médios da cobertura de nuvens cúmulos rasos para os experimentos $\mathrm{SHA}$ (sem interação entre a radiação e a convecção rasa) e RAD (com interação entre a radiação e a convecção rasa), para o regime de vento de oeste (doravante chamado de $\mathrm{W}_{2}$ ). A Figura $1 \mathrm{~b}$ mostra o mesmo para o regime de vento de leste (que doravante será chamado de $\mathrm{E}_{3}$ ). A cobertura de nuvem do modelo surge a partir das $7 \mathrm{~h}$ e termina

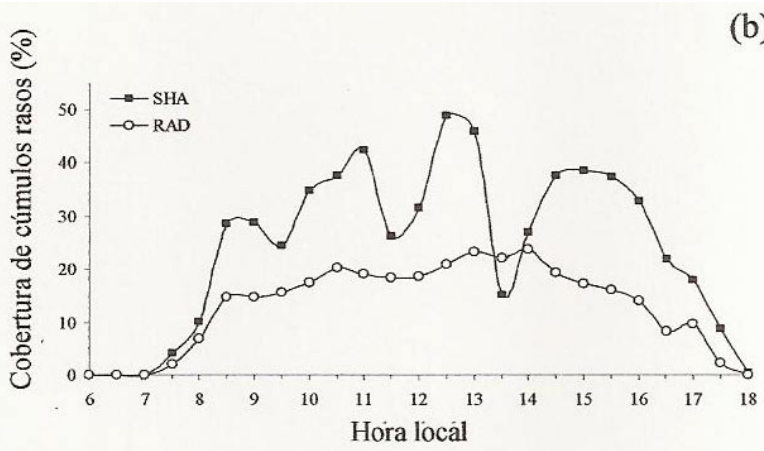

Figura 1 - Ciclo diurno médio da cobertura de cúmulos rasos para os experimentos SHA e RAD para o regime de vento de oeste (a) e leste (b). 
em torno das 17:30 h no $\mathrm{W}_{2}$ e meia hora depois no $\mathrm{E}_{3}$. A fração da cobertura máxima para os experimentos foi em torno de $44 \%$ e $49 \%$ para SHA e $17 \%$ e $24 \%$ para o RAD nos períodos $\mathrm{W}_{2}$ e $\mathrm{E}_{3}$, respectivamente. Observa-se variação na cobertura da nuvem nos dois experimentos sendo que a do experimento SHA é maior. A cobertura de nuvem cúmulos rasos é maior para o $\mathrm{E}_{3}$, assim como a duração, o que não é consistente com os resultados da cobertura de nuvens observada por Betts et al., (2002) que verificaram que o $\mathrm{W}_{2}$ apresentou cobertura de nuvens maior do que $\mathrm{E}_{3}$. Isso pode ser atribuído (ver discussão mais à frente) à condensação na escala da grade no $\mathrm{W}_{2}$ ser bem maior do que no $\mathrm{E}_{3}$ atenuando a radiação no $\mathrm{W}_{2}$ e consequentemente, diminuindo a cobertura de nuvem nesse regime. A diminuição no valor da cobertura de cúmulos rasos para metade entre os experimentos SHA e RAD representa uma melhoria no sentido de resultados mais realistas, conforme os mostrados para vários esquemas por Lenderink et al. (2004) e Grabowski et al (2006).

A Figura 2a mostra o ciclo médio do saldo de radiação para o $\mathrm{W}_{2}$. $\mathrm{O}$ saldo de radiação do modelo é igual ao observado somente nas primeiras horas da manhã. Após as 8 $\mathrm{h}$, a interação entre a radiação e a cobertura de cúmulos rasos produz um ciclo que subestima aquele sem a interação com a radiação e as observações, enquanto que o SHA é maior que o observado somente em alguns horários, e a partir das $15 \mathrm{~h}$ os dois experimentos são aproximadamente iguais. Observa-se na Figura 2b, que o ciclo médio do saldo de radiação para os experimentos $\mathrm{SHA}$ e RAD para o $\mathrm{E}_{3}$ tem seu início e término, meia hora antes do observado. No período da manhã, o modelo tende a superestimar o saldo de radiação observado e à tarde o experimento que mais se aproxima do ciclo observado é o SHA, com valores ligeiramente maiores para os dados observados. Porém, o saldo de radiação simulado com interação com a radiação tem variabilidade semelhante à dos dados observados. O fluxo máximo do saldo de radiação do experimento RAD acontece 2,5 horas antes do observado, e o do experimento SHA ocorre no mesmo horário. A discrepância de meia hora no início e final do ciclo diurno com relação às observações também foi observada por Souza e Silva Dias (2004), isso acontece porque as nuvens que ainda existem nas primeiras horas da manhã e no final da tarde são mal (quando não) representadas pelo modelo. A diferença de meia hora entre o início do ciclo do saldo de radiação simulado é atribuída à ordem com que as parametrizações são acionadas no modelo. Um aumento da radiação implica em um aumento simultâneo na cobertura de nuvem, que por sua vez, vai atenuar a radiação na próxima vez que ela for acionada, o que ocorre com freqüência de meia hora. O saldo de radiação do experimento SHA se correlaciona melhor com o observado do que o RAD, o que leva a crer que a cobertura de nuvem desse experimento é mais representativa da cobertura de nuvem observada, $W_{2}$. Vale ressaltar que o saldo de radiação do modelo é atenuado apenas pelos cúmulos rasos, visto que a parametrização foi efetuada somente para esse tipo de nuvem. Portanto, os efeitos de grande escala e convecção profunda não influenciaram no saldo de radiação do modelo.

O ciclo médio do fluxo de calor sensível do modelo e observado para o $\mathrm{W}_{2}$ é exposto na Figura 3a. Antes das $11 \mathrm{~h}$, o experimento SHA superestima o fluxo observado, enquanto o RAD tende a subestimá-lo. No período da tarde os fluxos tentem a apresentar valores semelhantes, com o experimento SHA acompanhando melhor a variabilidade dos dados observados. Já para o $\mathrm{E}_{3}$ (Figura 3b) o modelo superestima as observações até antes das 13 h. Neste caso, o experimento RAD diverge menos do observado. Outro ponto a ser observado, é que o experimento RAD tende a acompanhar melhor a variabilidade das observações, o que não ocorre com o SHA. Na parte final do ciclo, quando os valores diminuem, o experimento RAD passa a subestimar as observações, enquanto o SHA continua a superestimá-la. Contudo, o RAD continua a captar a variabilidade observada. No estudo de Betts e Jakob (2002), o fluxo de calor sensível do modelo foi maior que o observado
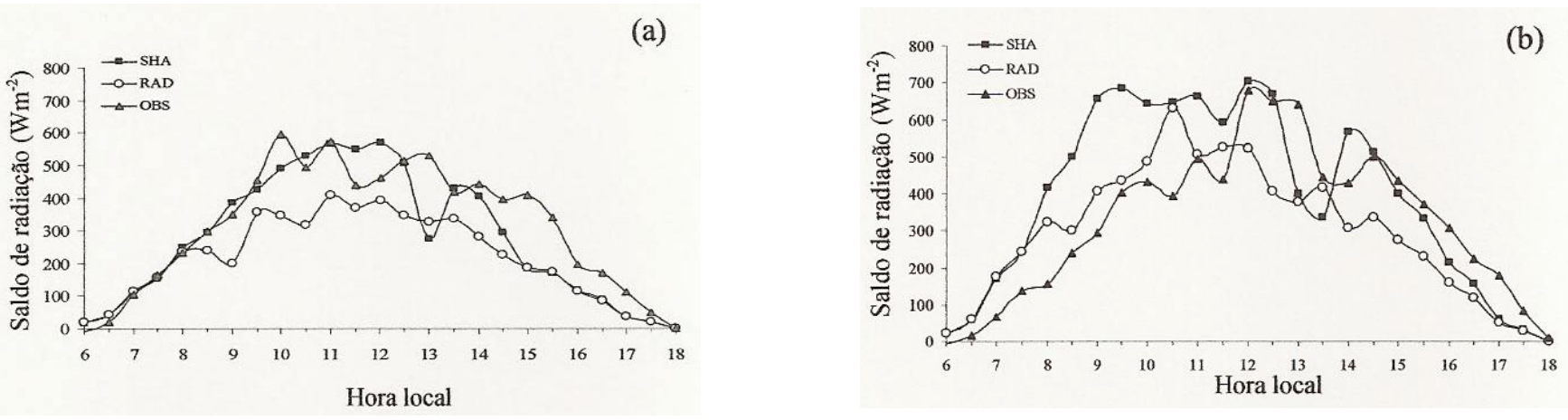

Figura 2 - Ciclo diurno médio do saldo de radiação para os experimentos SHA e RAD para o regime de vento de oeste (a) e de leste (b). 
o mesmo foi encontrado por Souza e Silva Dias (2004), mas para cobertura vegetal de pastagem.

De acordo com a Figura 4a, o ciclo de calor latente representado pelo modelo para $\mathrm{o} \mathrm{W}_{2}$ inicia-se aproximadamente uma hora e meia antes do observado. Essa diferença de tempo no começo do fluxo do modelo é refletida no horário em que ocorre o seu fluxo máximo, que acontece às $12 \mathrm{~h}$ e das observações, uma hora e meia após. Por causa da grande variabilidade no fluxo observado, no período da tarde, ora o fluxo do modelo é subestimado ora é superestimado. O fluxo de calor latente para o $\mathrm{E}_{3}$ é visto na Figura 4b. Percebe-se que no início e no final do ciclo diurno os fluxos dos dois experimentos têm comportamento semelhante, com valores ligeiramente menores para o experimento RAD, no final do ciclo diurno. No período da manhã, o modelo superestima o fluxo de calor latente observado e até por volta das $15 \mathrm{~h}$, o observado é subestimado pelo RAD e superestimado pelo SHA, com exceção das $14 \mathrm{~h}$, quando existe uma pronunciada queda no fluxo de calor latente do experimento
SHA. A interação entre a radiação e a convecção rasa faz com que os fluxos de calor latente do modelo sejam semelhantes aos das observações. O mais interessante é que a variabilidade que o experimento RAD apresenta é semelhante às observações, em alguns horários há aumento e queda simultâneos do fluxo de calor latente entre o experimento RAD e o observado, sendo isso mais acentuado para o $\mathrm{E}_{3}$. A discrepância vista no início e final do ciclo diurno dos fluxos também foi observada por Betts e Jakob (2002). Segundo os autores, ao amanhecer próximo da camada a superfície é geralmente saturada e estabilizada pelo resfriamento radiativo e pela precipitação do dia anterior. Os fluxos de calor sensível e latente são, inicialmente, confinados numa camada rasa estável com menos de $400 \mathrm{~m}$ de profundidade e a temperatura potencial e a razão de mistura inicialmente crescem rapidamente nessa camada rasa de mistura. $\mathrm{O}$ modelo representa isso razoavelmente bem, mas somente duas horas após o nascer do sol. Souza e Silva Dias (2004) também encontraram diferença no início do ciclo diurno no fluxo de calor (a)

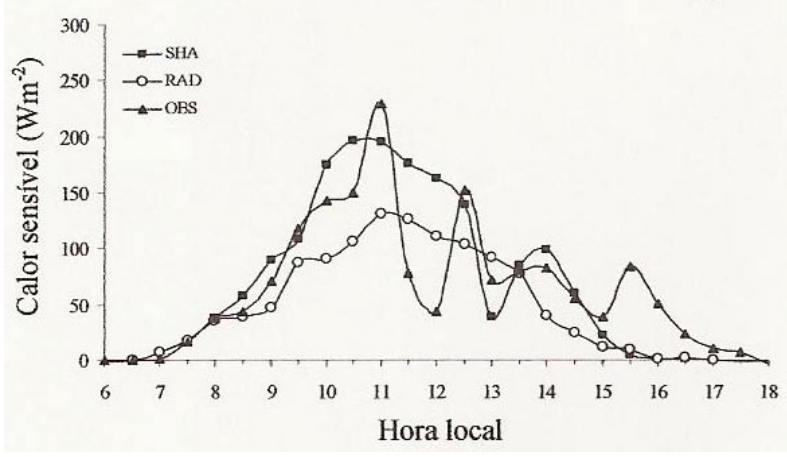

(b)

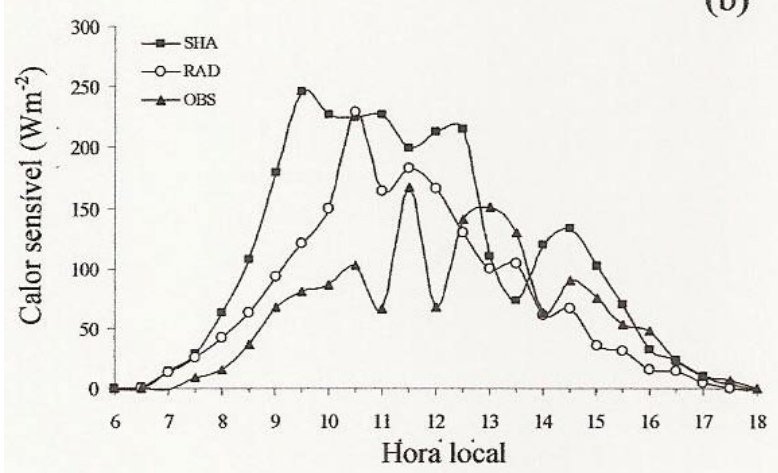

Figura 3 - Ciclo diurno médio do fluxo de calor sensível para os experimentos SHA e RAD e observado para o regime de vento de oeste (a) e de leste (b).
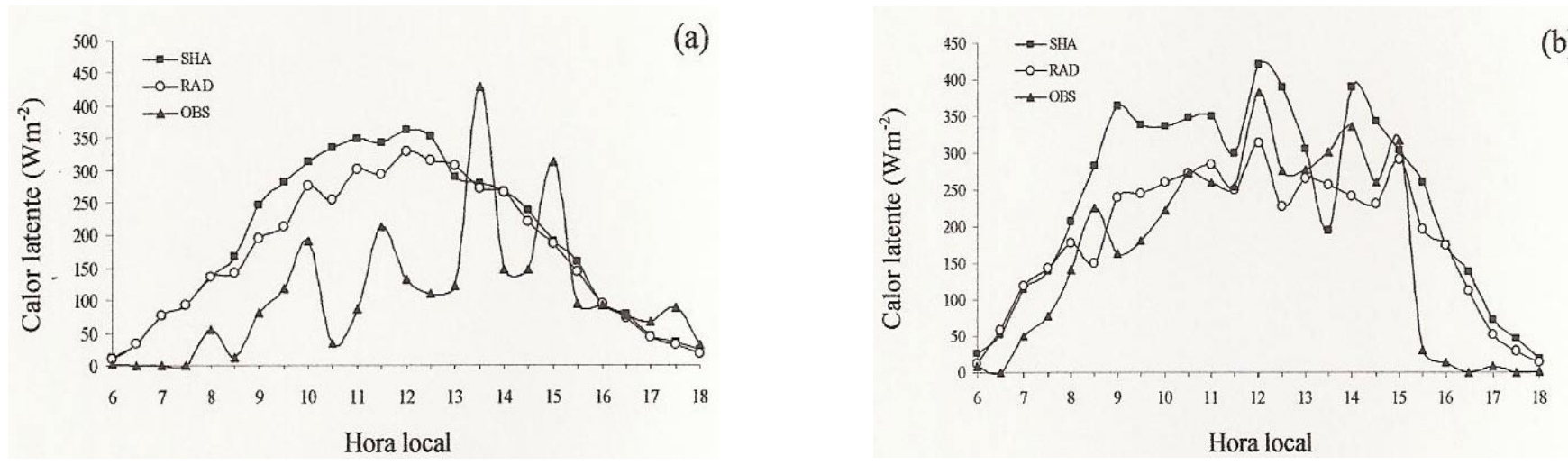

(b)

Figura 4 - Ciclo médio diurno do fluxo de calor latente para os experimentos SHA e RAD e observado para o regime de vento de oeste (a) e leste (b). 
latente, e apontaram que isso está relacionado à parte do modelo que mais reflete as imperfeições da representação dos fenômenos físicos durante a transição do período noturno para o diurno.

As frações evaporativas dos experimentos numéricos e a observada são apresentadas na Figura 6a para o $\mathrm{W}_{2}$. A fração evaporativa (FE) foi estimada de acordo com Betts e Jakob (2002) e é dada por $F E=L E /(L E+H), L E$ é o fluxo de calor latente e $\mathrm{H}$ é o fluxo de calor sensível. Verifica-se que há uma simetria entre os experimentos SHA e as observações durante todo o período. No início da manhã e após as 14:30 h, a FE do modelo utilizou mais energia na forma de calor latente do que na forma de calor sensível o que concorda com a Figura 4a. No E3, durante todo o período, o modelo subestima a FE observada, essa diferença é menor a partir das $14 \mathrm{~h}$, principalmente no experimento RAD devido a FE desse experimento ser superior a do SHA. Essa superestimativa pode ser atribuída ao fluxo de calor sensível observado que é muito baixo. O experimento com interação nuvem-radiação tende a acompanhar a variabilidade da
FE observada, o que se pode atribuir ao efeito do sombreamento da convecção rasa no balanço de energia.

Os ciclos diurnos da razão de mistura dos dois experimentos para o $\mathrm{W}_{2}$ (Figura 6a) tendem a acompanhar o comportamento da razão de mistura observada mostrando o crescimento da razão de mistura no inicio do dia. Aproximadamente 3 horas após a aurora, a razão de mistura decresce por causa do transporte ascendente do vapor d'água na camada de mistura para dentro do campo dos cúmulos rasos. Nas primeiras horas da manhã, o experimento SHA é um pouco mais úmido que o RAD e depois desse horário, ele torna-se mais seco. A razão de mistura do modelo é maior que a observada entre as primeiras três horas e após as $15 \mathrm{~h}$. Entre 9 e $14 \mathrm{~h}$ o modelo tende a subestimar a razão de mistura. $\mathrm{No} \mathrm{E}_{3}$ (Figura 6b) o comportamento do modelo representa razoavelmente bem a observação. Apesar da pequena diferença entre os experimentos SHA e RAD, pode-se verificar que este último apresenta uma variação que tende a representar melhor o ciclo observado.
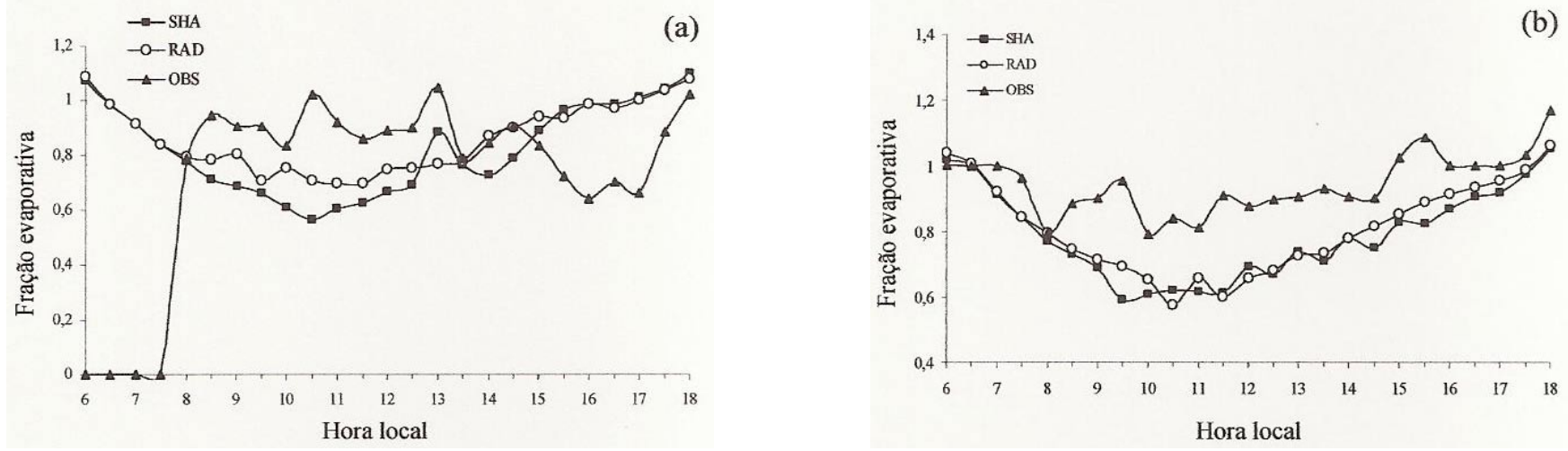

Figura 5 - Ciclo diurno médio da fração evaporativa para os experimentos SHA e RAD e observado para o regime de vento de oeste (a) e leste (b).
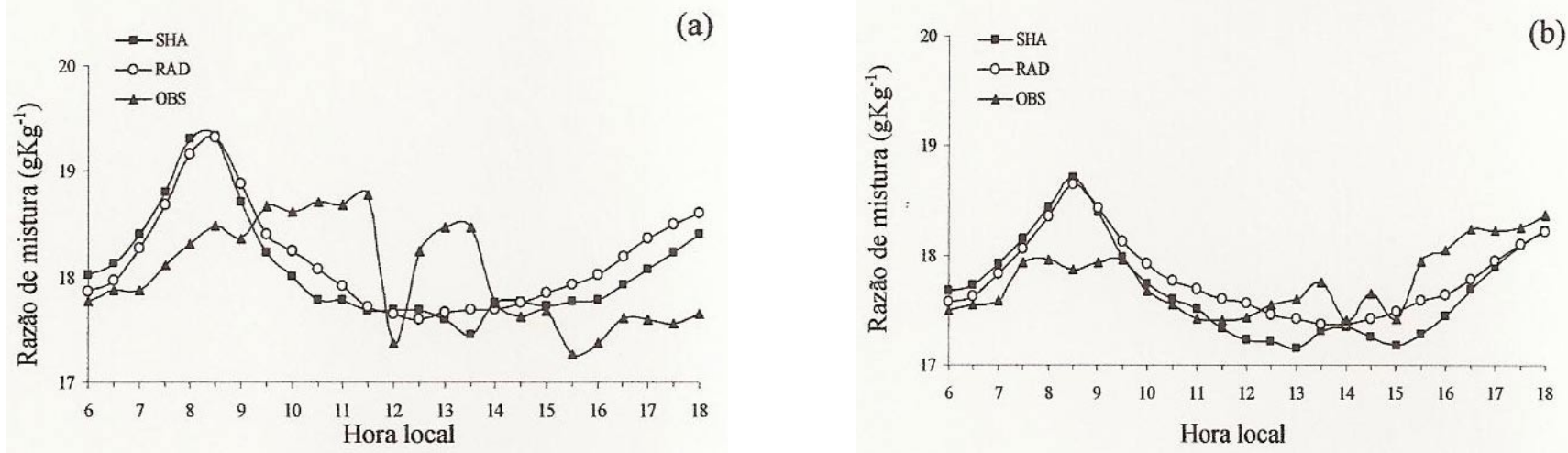

Figura 6 - Ciclo diurno médio da razão de mistura para os experimentos SHA e RAD e observado para o regime de vento de oeste (a) e leste (b). 
Analisando-se a temperatura potencial para $\mathrm{W}_{2}$ (Figura 7a), percebe-se que o modelo superestima a temperatura potencial observada por mais de $2 \mathrm{~K}$ nas primeiras horas. Ao longo do dia, essa diferença diminui. Entre 13 e 16 h, os valores simulados e observados são similares. Note-se que o experimento RAD tende a manter a temperatura ligeiramente inferior a do experimento SHA. A Figura $7 b$ mostra a temperatura potencial para E3. O aumento observado até as $11 \mathrm{~h}$ não é acompanhado pelo modelo. Após o meio-dia, as simulações seguem razoavelmente bem a observação.

A Figura 8a mostra a condensação devida à saturação na escala da grade para o $\mathrm{W}_{2}$. Isso serve para avaliar o impacto da configuração dos fluxos de superfície em algumas variáveis do modelo. A saturação na escala da grade é vista no modelo como a formação de uma nuvem estratiforme, que interagem com a radiação. $\mathrm{Na}$ ausência de convecção rasa, essa condensação é uma forma de atenuação da radiação e é muito importante em resolução nas quais os processos úmidos são mais bem representados pela parametrização da microfísica. $\mathrm{O}$ experimento $\mathrm{RAD}$ apresenta menor quantidade e duração de condensação que o experimento SHA. A condensação para o E3 é mostrada na Figura 8b. O experimento RAD praticamente não apresenta essa condensação, ao contrário do experimento SHA, que tem maior condensação em quantidade e duração. Isso tudo implica que, em resoluções nas quais a parametrização de microfísica não trabalha de forma efetiva, a parametrização de convecção rasa, interagindo com os processos radiativos, tende a produzir resultados mais realistas.

\section{CONSIDERAÇÕES FINAIS}

Este trabalho foi desenvolvido no intuito de averiguar o impacto da convecção rasa no ciclo diurno do balanço de energia à superfície na Amazônia. O enfoque foi dado no efeito de sombreamento dos cúmulos rasos na configuração do forçamento à superfície. O modelo BRAMS foi utilizado
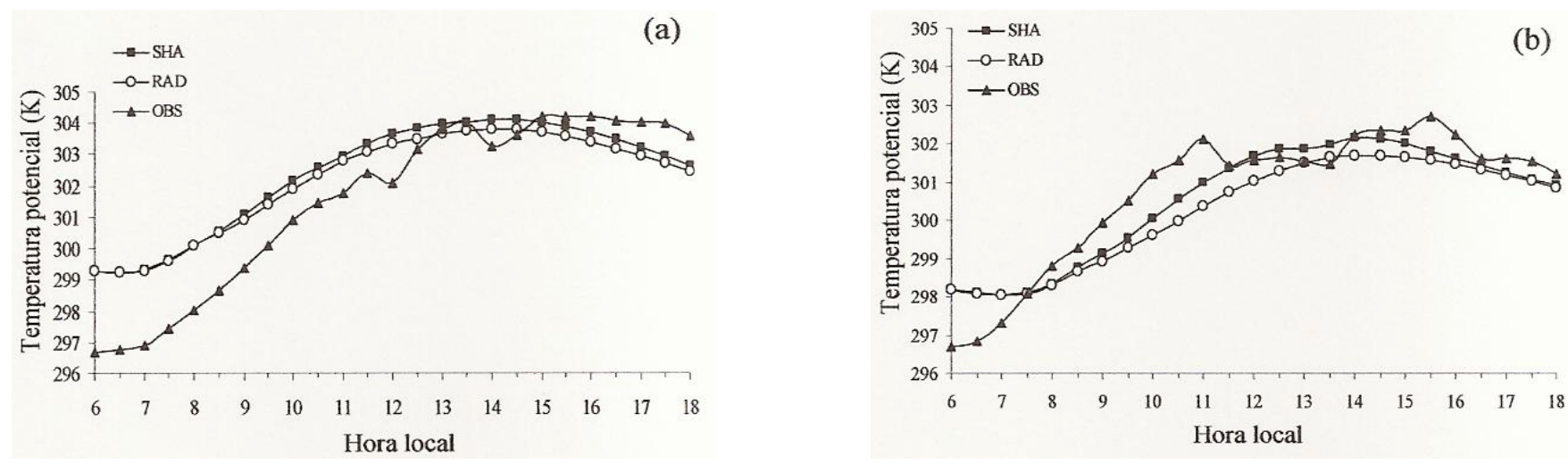

Figura 7 - Ciclo diurno médio da temperatura potencial para os experimentos SHA e RAD e observado para o regime de vento de oeste (a) e leste (b).
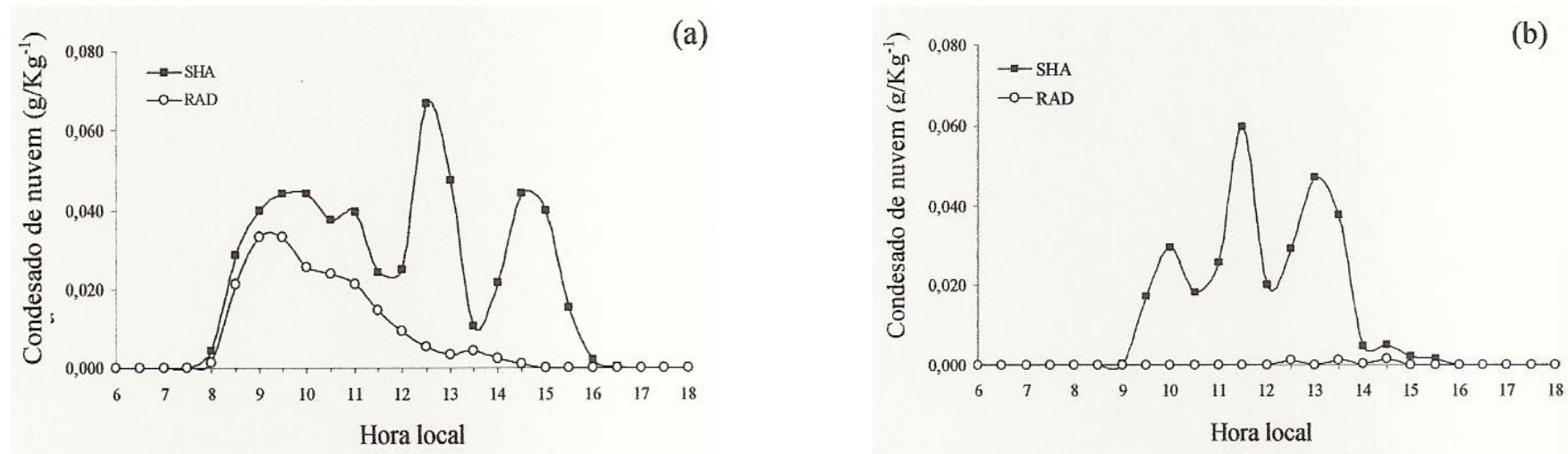

Figura 8 - Ciclo diurno médio da condensação na escala da grade para os experimentos SHA e RAD para o regime de vento de oeste (a) e leste (b). 
nas simulações dos fluxos à superfície, e foram realizados dois experimentos no período de atuação dos ventos de baixos níveis de oeste e de leste no estado de Rondônia.

O ciclo diurno dos fluxos à superfície foi bastante sensível à cobertura de cúmulos rasos. Isso foi mais pronunciado no período de atividade máxima dessas nuvens. De modo geral, a interação nuvem-radiação atuou no sentido de diminuir o forçamento convectivo e esses resultados apontam para uma melhoria na qualidade das simulações. Outro ponto importante reside no fato de as simulações com a interação conseguirem capturar a variabilidade dos fluxos de superfície. Isso é muito importante para uma representação mais realista da camada limite turbulenta.

Embora a interação cúmulos-radiação melhore o ciclo diurno dos fluxos à superfície, o modelo não conseguiu representar bem esses fluxos no início e final do ciclo diurno. Isso pode ser atribuído ao fato de a convecção noturna ser mal representada no modelo. Os cúmulos rasos que são gerados por turbulência mecânica antes do amanhecer, permanecem na atmosfera por algumas horas, como nuvens passivas, até serem substituídas por cúmulos formados por turbulência térmica. Como o modelo não consegue representar a interação dessas nuvens com a radiação, essa é uma possível causa para o forte aumento dos fluxos simulados no início do dia. Pesquisa voltada para a melhoria da simulação no início do dia é o tema da continuação deste trabalho.

\section{AGRADECIMENTO}

Parte deste trabalho foi realizada no período em que Zilurdes Fonseca Lopes era bolsista do curso de Pós-Graduação em Meteorologia na Universidade Federal de Campina Grande. Os autores agradecem ao CNPq pelo suporte financeiro.

\section{REFERÊNCIAS BIBLIOGRÁFICAS}

ALBRECHT, B. A. Parameterization of trade-cumulus cloud amounts. Journal of the Atmospheric Sciences: v.38, p.97105, 1981.

ARAKAWA, A.; SCHUBERT, W. H. Interaction of a cumulus cloud ensemble with the large-scale environment. Part I. Journal of the Atmospheric Sciences: v.31, p.674-701, 1974.

BETTS, A.K. Parametric interpretation of trade-wind cumulus budget studies. Journal of the Atmospheric Sciences: v.32, p.1934-1945, 1975.

BETTS, A. K.; MILLER, M. J., A new convective adjustment scheme. Part II: Single column tests using GATE wave, BOMEX,ATEX and arctic air-mass data sets. Quarterly Journal of the Royal Meteorological Society, v.112, p.693-709. 1986.
BETTS, A. K. et al. Surface diurnal cycle and boundary layer structure over Rondonia during the rainy season. Journal of Geophysical Research: v.107, N ${ }^{\circ}$.D20.8065, doi:10.1029/ 2001JD000356, 2002.

BETTS, A. K.; JAKOB, C. Evaluation of the diurnal cycle of precipitation, surface thermodynamics, and surface fluxes in the ecmwf model using LBA data. Journal of Geophysical Research: v. 107, Nº.D20.8045, doi:10.1029/2001JD000427, 2002.

CHEN, C.; COTTON, W. R. A One-dimensional simulation of the stratocumulus capped mixed layer. Boundary-Layer Meteorology: v.25, p.289-321, 1983.

DENG, A. J.; SEAMAN, N. L.; KAIN, J. S. A shallowconvection parameterization for mesoscale models. Part II: verification and sensitivity studies. Journal of the Atmospheric Sciences: v.60, p.57-78, 2003.

GRABOWSKI, W .W. et al. Daytime convective development over land: A model intercomparison based on LBA observations. Quarterly Journal of the Royal Meteorological Society, v.132, p.317-344. 2006.

HAIDEN, T. Generalization of Albrecht's cumulus cloud amount parameterization. Journal of the Atmospheric Sciences. v.53, p.3164-3167, 1996.

KHAIROUTDINOV, M.; RANDALL, D. Cloud-resolving modeling of the ARM summer 1997 IOP: Model formulation, results, uncertainties and sensitivities. Journal of the Atmospheric Sciences. v. 60, p. 607-625, 2003.

KUO, H. L. Further studies of the parameterizations of the influence of cumulus convective on large-scale flow. Journal of the Atmospheric Sciences: v.31, p.1232-1240, 1974.

LENDERINK, G. et al. The diurnal cycle of shallow cumulus over land: A single-column model intercomparison study. Quarterly Journal of the Royal Meteorological Society, v.130, p.3339-3364. 2004.

LIOU, K-N. An introduction to atmospheric radiation. New York: Academic Press. 392p., 1980.

MELLOR, G. L.; YAMADA, T. Development of a turbulence closure model for geophysical fluid problems. Reviews of Geophysics and Space Physics: v.20, p.851-857, 1986.

MOLINARI, J.Ageneral form of Kuo's cumulus parameterization. Monthly Weather Review. v. 113, p.1411-1416, 1985.

MOLINARI, J.; CORSETTI, T. Incorporation of cloud-scale and mesoscale downdrafts into a cumulus parametrization: results of one - and three-dimensional integrations. Monthly Weather Review, v.113, p.485-501, 1985.

PEREIRA, G.; RUTLEDGE, S. Diurnal cycle of shallow and deep convection for a tropical land and an ocean environment and its relationship to synoptic wind regimes. Monthly Weather Review, v.134, p.2688-2701, 2006.

PIELKE, R. A. et al. A comprehensive meteorological modeling 
system-RAMS. Meteorology and Atmospheric Physics. v. 49, p.69-91, 1992.

RENNÓ, N. O.; INGERSOLL, A. P. Natural convection as a heat engine: a theory for CAPE. Journal of the Atmospheric Sciences. v. 53, p.572-585, 1996.

RENNÓ, N. O.; WILLIAMS, E. R. Quasi-Lagrangian measurements in convective boundary layer plumes and their implications for calculation of CAPE. Monthly Weather Review. v. 123, p.2733-2742, 1995.

RICKENBACH, T. M. et al. Mesoscale properties of convection in western Amazonia in the context of large-scale wind regimes. Journal of Geophysical Research.v 107, 10.1029/ 2000JD000263, 2002.

SIEBESMA, A.P. et al. A Large Eddy Simulation intercomparison study of shallow cumulus convection. Journal of the Atmospheric Sciences: v. 60 n.10, p. 1201-1219, 2003.

SILVA DIAS, M. A. F. et al. Clouds and rain processes in a biosphere-atmosphere interaction context in the amazon region: Journal of Geophysical Research. v.107. $\mathrm{N}^{\circ}$. D20.8072, doi:10.1029/2001JD000335, 2002.

SOUZA, E. P. Estudo teórico e numérico da relação entre convecção e superfícies heterogêneas na região amazônica. 1999. 121 p. Tese (Doutorado em Meteorologia) Universidade de São Paulo, São Paulo, 1999.
SOUZA, E. P.; SILVA, E. M. Impacto da implementação de uma parametrização de convecção rasa em um modelo de mesoescala: Descrição e testes de sensibilidade do esquema. Revista Brasileira de Meteorologia, v.18, 33-42. 2003.

SOUZA, E. P.; SILVA DIAS, P. L. Interação entre nuvens convectivas não precipitantes e radiação. In: XIII Congresso Brasileiro Meteorologia, 2004. Fortaleza. Anais... Fortaleza, 2004. CD-ROM.

STULL, R. B. A fair-weather cumulus cloud classification scheme for mixed-layer studies. Journal of Climate and Applied Meteorology., v.24, p. 49-56, 1985.

TIEDTKE, M. A comprehensive mass flux scheme for cumulus parameterization in large-scale models. Monthly Weather Review, v.117, 1779-1800. 1989.

WILDE, N. P.; STULL, R. B.; ELORANTA, E. W. The LCL zone and cumulus onset. Journal of Climate and Applied Meteorology. vol. 24, 640-657, 1984

WALKO, R. L. et al. New rams cloud microphysics parameterization. 1. The Single-Moment Scheme. Atmospheric Research: v.38, p.29-62, 1995.

WALKO, R. L. et al. Coupled atmosphere-biophysics-hydrology models for environmental modeling. Journal of Applied meteorology. v. 39, p. 931-944, 2000. 